\title{
La poly(ADP-ribose) polymérase (PARP) : à la croisée des chemins de la réparation de l'ADN et de l'apoptose cellulaire
}

La cellule est constamment exposée à des agents génotoxiques provenant de l'environnement: quand la molécule d'ADN est atteinte, elle est aussitôt réparée pour maintenir l'intégrité du génome. Le bris de l'un ou des deux brins de l'ADN constitue la lésion la plus grave, aussitôt signalée à la cellule par la stimulation de l'activité d'une enzyme, la poly(ADP-ribose) polymérase (PARP). Cette enzyme nucléaire, abondante chez les eucaryotes supérieurs, catalyse la formation d'un polymère d'ADP-ribose sur des accepteurs nucléaires tels la PARP (automodification), les topoisomérases et les histones, altérant ainsi les fonctions de ces protéines $[1,2]$. Depuis une quinzaine d'années, des résultats suggéraient, de façon indirecte, un rôle de la PARP dans la réparation de l'ADN [1, 2]. Récemment, une démonstration directe de ce rôle a été faite en utilisant un test in vitro de réparation de l'ADN: un plasmide contenant une seule coupure est réparé par un extrait cellulaire. Cette réparation in vitro est dépendante du $\mathrm{NAD}^{+}$et influencée par la PARP $[3,4]$. La poursuite de ces études, utilisant l'ADN endommagé par divers types d'agents, a permis de montrer que la PARP jouait principalement un rôle dans la réparation par excision des bases (REB) de l'ADN et non dans la réparation par excision des nucléotides (REN) [3-6]. En outre, plusieurs études in vivo ont indiqué que, après lésion de l'ADN, l'activité de la REB requiert la PARP: ainsi, l'inhibition transdominante de la PARP par la surexpression de son domaine de liaison à l'ADN démontre le rôle de la PARP dans la REB lorsque l'ADN est endommagé par les agents alky- lants et non par les rayons ultra-violets $[5,6]$.

Lorsque les dommages à l'ADN sont trop sévères, la cellule entre en apoptose sous l'action de p53. Ce phénomène active la PARP. Les mécanismes moléculaires de l'apoptose, phénomène de transformation morphologique de la cellule qui est en voie de mort cellulaire programmée (et non nécrotique), demeurent mal compris. Parmi les différents changements biochimiques survenant dans la cellule en apoptose, les activités protéasiques sont de première importance [7]. L'interleukine-1 $\beta$ converting enzyme (ICE), un homologue de la protéine codée par ced-3, un gène de mort cellulaire chez le nématode C. elegans ( $m / s n^{\circ} 2$, vol. 10, p. 232), est une protéase qui induit l'apoptose ( $m / s n^{\circ} 7$, vol. 11, p. 1053) [8]. Cependant, il y a plusieurs indications que toutes les voies aboutissant à l'apoptose ne passent pas par ICE. En effet, l'apoptose des thymocytes et des macrophages est normale chez des souris déficientes en ICE. De plus, l'apoptose est également associée à un clivage de la PARP au niveau du résidu aspartyl en position 216 , ce qui ne correspond pas à la spécificité de l'ICE. Les équipes de Earnshaw, Poirier et Kaufmann avaient montré que la PARP extraite de cellules en mitose était clivée spécifiquement par une protéase analogue à ICE, la prICE, et que l'inhibition de cette protéase empêchait l'apoptose [9] ; le même phénomène se produisait dans les cellules HL60 [10]. En fait, il existe toute une famille de "protéases apoptotiques" dont fait aussi partie la protéase Yama/CPP32B, appartenant à la famille ICE et plus proche qu'elle de la protéine Ced-3. L'activation de cette dernière par ICE entraîne une cascade d'événements qui enclenchent la mort cellulaire: Yama/CPP32B protéolyse la PARP (116 kDa) au début du processus apoptotique, libérant ainsi deux fragments de $89 \mathrm{kDa}$ et 24 kDa dans la cellule [11]; la détection du fragment 89 kDa est considérée comme un indicateur précoce de l'apoptose et le fragment de $24 \mathrm{kDa}$ contient une partie du domaine de liaison à l'ADN (doigts de zinc) de PARP; ce fragment agirait comme un inhibiteur de la PARP intacte, ce qui enclencherait un signal de début de réparation de l'ADN. Par ailleurs, l'inhibition de la protéase Yama/CPP32 $\beta$ par la protéine CrmA [12] inhibe complètement l'apoptose ainsi que l'hydrolyse de la PARP. On a aussi suggéré d'autres rôles pour la PARP dans l'apoptose: (1) l'activation de la PARP à la suite d'un dommage de l'ADN peut causer l'apoptose par déplétion des réserves de $\mathrm{NAD}^{+}[1,2]$ et, par ricochet, d'ATP; (2) les besoins énergétiques du processus apoptotique peuvent être contrôlés par la protéolyse de la PARP [10]. ICE et CPP-32 sont synthétisées sous la forme de proenzymes qui doivent être activées par une coupure de maturation protéolytique. On ne connaît pas les enzymes responsables de cette activation; existe-t-il une cascade de protéases à cystéine de la famille de ICE, incluant la CPP-32, s'activant l'une l'autre avant d'aboutir au clivage de cibles qui serait alors responsable de l'apparition des signes caractéristiques de l'apoptose ? $\left(\mathrm{m} / \mathrm{s} n^{\circ} 10\right.$, vol. 11, p. 1502). L'intérêt de la découverte des membres de cette famille de protéases "apoptotiques" est théorique, mais aussi potentiellement
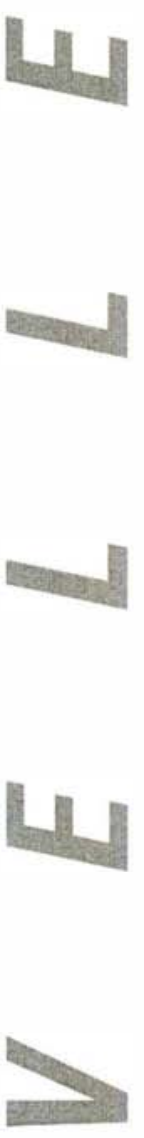
pratique: les inhibiteurs de ces enzymes, comme cela a déjà été démontré pour ICE $\left(m / s n^{\circ} 7\right.$, vol. 11, $p$. 1053), et confirmé pour Yama/ CPP32 $\beta$, sont de potentielles molécules protectrices contre l'apoptose. Des chercheurs de la compagnie Merck ont récemment purifié la protéase CPP32 et leurs résultats confortent bien le modèle selon lequel cette protéase agit sur la PARP dans la cascade des événements reliés à l'apoptose [13].

S.G.

S.D.

W.E.

S.K.

G.G.P.

*ABRÉVIATIONS*
REB: réparation par excision des bases
REN: réparation par excision de nu-
cléotides
ICE: interleukin $1 \beta$ converting en-
zyme
PARP: poly(ADP-ribose) polymérase
prICE: protease resembling ICE.

1. Althaus FR, Richter C. ADP-ribosylation of proteins. New York: Springer V'erlag, 1987.

2. Lautier D, Lagueux J, Thibodeau J, Ménard L Poirier GG. Molecular and biochemical features of poly(ADP-ribose) metabolism. Mol Cell Biochem $1993 ; 122$ : 171-93.

3. Satoh MS, Lindahl T. Role of poly(ADP-ribose) formation in DNA repair. Nature 1992; 356: 356

4. Satoh MS, Poirier GG; Lindahl T. NAD+dependent repair of damage DNA by human cell extracts. J Biol Chem 1993; 268: 5480-7.

5. Molinete M, Vermeulen W, Burkle A, Ménis sier-de Murcia J, Kupper JH, Hoeijmakers JHJ, de Murcia (;. Overproduction of the poly(ADP-ribose) polymerase DNA-binding domain blocks alky lation-induced DNA repair synthesis in mammalian cells. EMBO J 1993; 12: 2109-17.

6. de Murcia (; Ménissier de Murcia J. Poly(ADPribose) polymerase: a molecular nick sensor Trends Biochem Sci 1994; 19: 172-6.

7. Vaux DL, Haecker (;, Strasser A. An evolutionary perspective on apopt osis. Cell 1994; 76 : 777 9.

8. Miura M, Zhu H, Rotello R, Hartweig EA, Yuan J. Induction of apoptosis in fibroblasts by IL-IB- converting enzyme, a mammalian homolog of the C.elegans cell death gene ced-3. Cell 1993; 75:653-60). 9. Lazebnik YA, Kaufmann SH, Desnoyers S, Poirier GC; Earnshaw WC. prICE, an ICE-like proteinase from apoptotic cells, cleaves poly(ADP-ribo se) polymerase. Nature $1994 ; 371: 346-7$.

10. Kaufmann S, Desnoyers S, Ottaviano Y, Davidson NE, Poirier GG. Specific proteolytic cleavage of poly(ADP-ribose) polymerase: an early marker of chemotherapy-induced apoptosis. Cancer Res $1993 ; 53: 3976-85$

11. Martinou J. La mort cellulaire programmée dans le système nerveux. médecine/sciences 1995 11: 367-73

12. Tewari M, Quan LR, O'Rourke K, Desnoyers S, Zeng Z, Deidler DR, Poirier GG; Salvesen GS, Dixit VM. Yama/C.PP32 $\beta$, mammalian homolog of CED-3, is a CrmA-inhibitable protease that cleaves the death substrate poly(ADP-ribose) polymerase. Cell 1995; $81: 801-9$.

13. Nicholson D, Ambereen A, Thornberrey NA Vaillancourt, JP, Ding CK, Gallant M, Gareau Y, Griffin PR, Labelle M, Lazebnik YA Munday NA Raju SM, Smulson ME, Yamin T, Yu VL, Miller $\mathrm{DK}$ Identification and inhibition of the ICE/CED-3 protease necessary for mammalian apoptosis. Nature $1995 ; 376: 37-43$.

\section{BRÈVES}

Polymorphisme du gène de l'enzyme de conversion de l'angiotensine (ACE) : à propos du cour et du rein. Cambien et al. (Inserm, SC7, Paris) ont établi l'association entre le génotype DD du gène de l'ACE et l'infarctus du myocarde, chez l'homme âgé de 25 à 64 ans et également chez le diabétique non-insulinodépendant [1]. Dans une étude coopérative avec des diabétologues danois [2], ils ont montré qu'il en était de même chez le diabétique insulinodépendant avec néphropathie ; la prévalence de la maladie coronaire est plus élevée en cas de néphropathie ( $19 \%$ contre $8 \%$ ). Chez les diabétiques avec néphropathie, la fréquence de la maladie coronaire est d'environ $20 \%$ en cas de génotype DD ou ID alors qu'elle n'est que de $7,5 \%$ en cas de génotype II. La distribution des génotypes est identique dans les deux groupes de diabétiques, avec ou sans néphropathie. Le même groupe de chercheurs a en effet montré qu'il n'y avait pas de relation entre le génotype de l'ACE et le risque de néphropathie et de rétinopathie proliférative [3], contrairement à une étude antérieure faite à Angers $\left(\mathrm{m} / \mathrm{s} n^{\circ} 4\right.$, vol. 10, p. 471). Cependant, les diabétiques avec néphropathie avaient un taux plasmatique d'ACE plus élevé que ceux indemnes de néphropathie. Nomura et al. au Japon ont démontré une liaison entre les génotypes DD et ID et le risque d'infarctus du myocarde, dans un groupe de malades hémodialysés chroniques. La prévalence du génotype II est plus élevée chez les Japonais que chez les Européens [4]. Enfin, dans la plus fréquente des maladies glomérulaires chroniques primitives, la glomérulonéphrite à dépôts intercapillaires d'IgA, une étude écossaise a mis en évidence que chez les sujets ayant l'allèle D (DD ou ID), la maladie débute apparemment plus tard et requiert la dialyse plus précocement. Le rythme de progression de l'atteinte rénale, appréciée par l'inverse de la créatininémie au cours du temps, est plus rapide chez les malades DD [5].

[1. Cambien F, Soubrier F. médecine/sciences $1992 ; 8$ : 989-91.]

[2. Tarnow L, et al. Diabetologia 1995; $38: 798-803$.

[3. Tarnow L, et al. Diabetes 1995 ; 44 : 489-94.]

[4. Nomura $\mathrm{H}$, et al. Lancet 1994; 343 : 482-3.]

[5. Harden PN, et al. Lancet 1995; 345 : 1540-2.] 